TP Periodica Polytechnica Chemical Engineering

\author{
62(3), pp. 323-335, 2018 \\ https://doi.org/10.3311/PPch.11412 \\ Creative Commons Attribution (i)
}

RESEARCH ARTICLE

\section{Improvement of the Graphene Oxide Dispersion Properties with the Use of TOPSIS Based Taguchi Application}

\author{
Bariş Şimşek $^{1^{*}}$, Gözde Ultav ${ }^{2}$, Haluk Korucu ${ }^{1}$, Ahmet Yartaşi ${ }^{1}$
}

Received 23 August 2017; accepted after revision 12 December 2017

\begin{abstract}
The stability of the graphene oxide dispersions is an important issue in the preparation of medicine, printed flexible electronics, 3D printers and conductive inks. In order to improve the stability; mean and standard deviation of particle size, polydispersity index, zeta potential and conductivity of graphene oxide dispersion were selected as the main stability properties. The improvement rate between the estimate and the optimal conditions were calculated for the mean and standard deviation of particle size, polydispersity index, zeta potential and conductivity as $264.0 \%, 1875.0 \%, 583.3 \%, 5.0 \%$ and $50.0 \%$, respectively in terms of the GO quality characteristics. The improvement rate between the estimate and the optimal conditions were calculated for the mean and standard deviation of particle size, polydispersity index, zeta potential and conductivity as $42.7 \%$, $79.7 \%,-5.0 \%, 9.9 \%$ and $-86.7 \%$, respectively in terms of the GO quality characteristics. The result show that TOPSIS based Taguchi optimization in this study is effective to improve the graphene oxide dispersion stability.
\end{abstract}

\section{Keywords}

graphene oxide dispersion, Hummers method, stability, TOPSIS based Taguchi optimization, product design

\footnotetext{
${ }^{1}$ Department of Chemical Engineering, Faculty of Engineering, Çankırı Karatekin University, 18100, Uluyazı, Çankırı, Turkey

${ }^{2}$ Department of Nanotechnology and Nanomedicine, Graduate School of Science and Engineering, Hacettepe University, 06800, Ankara, Turkey

*Corresponding author, e-mail: barissimsek@karatekin.edu.tr
}

\section{Introduction}

Graphene oxide (GO), which has the supreme physical and chemical properties, is one of the most promising additive in terms of excellent dispersion stability, cost-effective potential, large-scale production of graphene-based materials [1-3]. By means of these features; GO has a wide range of applications such as functional fluids [4], solar cells [5-6], polymer composites [7], cement composites [8], drug delivery systems [9], conductive films [10], biosensors [11], transistors [12], super capacitors [13], nano composites [14], bio-materials [15], lithium ion battery [16], water treatment process [17], conductive polymers [18-19] and conductive inks [20]. In many industrial applications, the stability of graphene oxide dispersions plays a crucial role for the proper solvent preparation. Therefore, many researchers have done the studies to understand the dispersion behavior and to improve the dispersion stability. Konios et al. [21] prepared GO and reduced graphene oxide (rGO) dispersions with the different solvents and they showed that the GO and rGO samples forms a stable dispersion with the deionized-water, ethylene glycol and N-methyl-2-pyrrolidone (NMP). Taha-Tijerina et al. [22] prepared a dispersion with the deionized-water, ethylene glycol, ethanol and mineral oil and they illustrated that the GO samples forms a strong stable dispersion with the deionized-water and ethylene glycol. Graphene oxide-deionized water nano-fluids are more attractive options because of the formation of fairly strong stable dispersions with graphene oxide in the deionized water and the elimination of toxic solvents such as NMP.

The quality of the dispersions including "strong stability" can be represented with some criteria such as zeta potential value, thermal and electrical conductivity, $\mathrm{pH}$, and particle size. Therefore, many studies have been done to analyze the GO dispersion properties. The average particle size and zeta potential value [22], viscosity [23], pH [24], shear rheology [25], specific surface area [26] and thermal conductivity [3;27]were analyzed and the dispersion stability [22], the thermal conductivity [28], thermal performance [29] were analyzed without using any systematic analyzing such an experimental design approach. It has been determined that oxidants and $\mathrm{pH}$ of the 
solution were effective on the graphene oxide stability [22]. The effect of the components of the graphene oxide samples on the stability criteria has not been systematically analyzed with the use of experimental design approach. However, the systematical methods especially experimental design approach may be useful to understand the dispersion behavior.

The studies related to the graphene oxide dispersions have focused generally on one or two criteria. For example; Obreja et al. [30] prepared the graphene oxide dispersion in the deionized water and determined the size of particle on average as 102 $\mathrm{nm}$ and the standard deviation of particle's size as $29.1 \mathrm{~nm}$ with the device of particle size analyzer. Taha-Tijerina et al. [22] determined one of the most stable graphene oxide dispersions as the deionized water and they determined the size of particle on average as $110 \mathrm{~nm}$ and the zeta potential as $-113.77 \mathrm{mV}$ with the use of acoustic spectrometer. Konios et al. [21] analyzed the maximum solubility of graphene oxide and reduced graphene oxide in an inorganic solvent with the distilled water. Zhang et al. [27] prepared the dispersion of reduced graphene oxide which was synthesized by Hummers method with the ionized water and they determined the dispersion zeta potential's value as $-50.9 \mathrm{mV}$ and the thermal conductivity value as $0.86 \mathrm{~W} / \mathrm{m} * \mathrm{~K}$ of the dispersion. The following manner restricts the industrial use of graphene oxide dispersions that their one or two properties are separately improved as the zeta potential or particle's size. Thus, the simultaneous optimization of these two values with the multi-response optimizing techniques may help in obtaining more stable and useful graphene oxide dispersions and increase its usage rate in the manufacturing industry.

Aqueous graphene oxide applications such as 3D printing technology, nano composites manufacturing or thin film technology require the stable and homogeneous dispersions. So the factor effects on the product stability must be understood and the mixture ratios should be optimized to minimize the product variance. The goals for the optimization of the mixture proportions in this study can be summarized as follows: Firstly, it is aimed to determine if the factors, mixture proportions and experimental conditions are effective or not on $\mathrm{GO}$ and $\mathrm{rGO}$ dispersion properties, secondly to improve the quality of the suspensions by decreasing the variability of the stability with the use of multi-response optimization methodology.

This study recommends a novel approach which aims the improvement of graphene oxide and the reduced graphene oxide dispersions' quality criteria with the use of multi-response optimization techniques. The main contribution of study is to optimize the particle's size, zeta potential, polydispersity index graphene oxide properties and also the standard deviation of particle's size with the method of TOPSIS-based (Technique for Order Preference by Similarity to an Ideal Solution) Taguchi. Thus, it would be possible to produce the homogeneous graphene oxide dispersion at the large scales, to minimize the standard deviation and to improve the product quality. Another goal of this study aims to determine on the mixture ratios which optimize the stability properties of graphene oxide dispersions.

\section{Experimental}

\subsection{Graphene oxide synthesis}

Graphite $(<20 \mu \mathrm{m}$ from Sigma Aldrich and $<50 \mu \mathrm{m}$ from Merck), sulphuric acid (95-97\%, Merck) and sodium nitrate (99.99\% extra pure, Merck) has been stirred at $5^{\circ} \mathrm{C}$ for a several hours and then the potassium permanganate $(\mathrm{pH} 7-9$, Merck) has been added the mixture with regard to Hummers and improved Hummers method [2, 31-33]. The deionized water has been added to the suspension and the reaction temperature has been raised to $98^{\circ} \mathrm{C}$ as a result of the hydration heat. The oxidation process has been terminated with the addition of deionized water and hydrogen peroxide $(30 \% \mathrm{v} / \mathrm{v}, 10$ $\mathrm{ml}$ ). The graphene oxide powders have been purified via washing with hydrochloric acid solution and centrifuge application. GO has been obtained by drying wet solid product in oven for 12 hours. The reducing agent has been added while the graphene oxide suspension has been stirred and the suspension has been heated under the atmospheric pressure or pressurized reactor (under 6 bar pressure) for several hours. The reduced graphene oxide samples have been separated by the centrifugation and filtration, have been washed with the use of acetone and have been dried in the non-vacuum or under the vacuum containers for 24 hours. The experimental details for each runs have been added to Table 2 .

\subsection{Graphene oxide characterization}

Raman spectroscopy analyses were carried out by the Thermo DXR Raman device in Namık Kemal University Central Research Laboratory. Fourier Transform Infrared Spectroscopy (FTIR) analyses were implemented in the Çankırı Karatekin University with the use of Bruker Tensor II brand device. The Malvern zeta sizer was used to determine the average particle size of the GO and rGO samples.

\subsection{Dispersions of $\mathbf{G O}$ and $\mathrm{rGO}$}

GO and rGO dispersions have been prepared as the following. $3 \mathrm{mg}$ GO has been weighed and distributed in $4 \mathrm{~mL}$ water using ultrasonic probe. This suspension has been put into the zeta sizer tub. Zeta potential of GO dispersions has been measured by potential dip cell. 30mg RGO has been weighed and dispersed in $5 \mathrm{~mL}$ water with the use of Ultrasonic Probe $(60 \%$ amplitude, on ice, 10min). $50 \mu$ of this suspension has been taken, placed in a zeta sizer tub and completed with water (about $3.5 \mathrm{ml}$ ). Zeta potential of rGO dispersions has been measured by the potential dip cell after the Vortex mixing. 


\subsection{A general outlook on the issue of TOPSIS based Taguchi optimization}

The Taguchi method is one of the most effective methods to keep the quality criteria at demanded value and is used to determine the optimal mixture ratios to minimize the raw material utilization. Its most important advantage is to decrease the number of experiments as possible as with the use of orthogonal arrays [34]. The orthogonal array is expressed as $L_{a}\left(b^{c}\right)$, a where is the number of factor combination in the experiment, $\mathrm{b}$ is the number of level for factors and $\mathrm{c}$ shows the number of factors which are used in the experiments [34]. Taguchi method proposed that the signal to noise ratio $(\mathrm{S} / \mathrm{N})$ can be used to measure the quality characteristic deviates from the desired value [35-36]. These quality characteristics are defined for the stable dispersions as smaller the better (average particle size), the higher the better that should be maximized (electrical conductivity) or the nominal the better that should be kept a target value [37]. The variability degree of factors' effect on the quality characteristics could be analyzed efficiently by the regression analysis [38]. Moreover, $\mathrm{S} / \mathrm{N}$ ratios can be used in the multi-criteria decision matrix for TOPSIS application. As it is known, the Taguchi method is used only in the improvement of single performance response on its own. The TOPSIS methods which are mostly preferred with the method of Taguchi are the multi-criteria decision which takes the methods for the solution of multi-response optimization problems.

The $\mathrm{S} / \mathrm{N}$ ratio which can be the-smaller-the-better (Eq. (1)) or the-larger-the-better (Eq. (2)) for responses, $(\eta)$ is a useful tool to obtain significant factors by evaluating the minimum variance [39].

$$
\begin{aligned}
& \eta_{i j}=-10 \log _{10}\left[\frac{1}{n} \sum_{k=1}^{n} y_{i j k}^{2}\right] \\
& \eta_{i j}=-10 \log _{10}\left[\frac{1}{n} \sum_{k=1}^{n} \frac{1}{y_{i j k}^{2}}\right]
\end{aligned}
$$

$\eta_{i j}$ is the $\mathrm{S} / \mathrm{N}$ ratio for the response $j$ of experimental number $i$, and $y_{i j k}$ is the experiment result for the response $j$ of the experiment $i$, in the $k$ th replication; $n$ is the total number of replications [39]. The multiple-response problem can be easily converted to a single-response problem with the TOPSIS methodology steps such as the determination of the decision matrix, calculation of normalized ratings, identification of positive ideal and negative ideal solutions $\left(\mathrm{A}^{*}\right.$ and $\left.\mathrm{A}^{-}\right)$, calculation of the separation measures and the calculation of the ranking scores $\left(\mathrm{C}_{i}^{*}\right)$ [39]. The main concept of TOPSIS method is to choose the alternative solution which is at the closest distance to the ideal solution and at the farthest distance from the negative-ideal solution in the sense of geometry. The implementation of the TOPSIS-based Taguchi method does not need complex mathematical calculation with non-linear object and constraint functions. The details of the TOPSIS based Taguchi application steps can be found in Şimşek et al. [40].

\section{Proposed Methodology}

An 8-step methodology has been followed to improve the dispersions of the GO and rGO stability (Fig. 1). $\mathrm{L}_{18}\left(2^{1 * 3^{7}}\right)$ orthogonal array has been selected to improve graphene oxide dispersion properties such as the average particle size, standard deviation of the particle size, polydispersity index, zeta

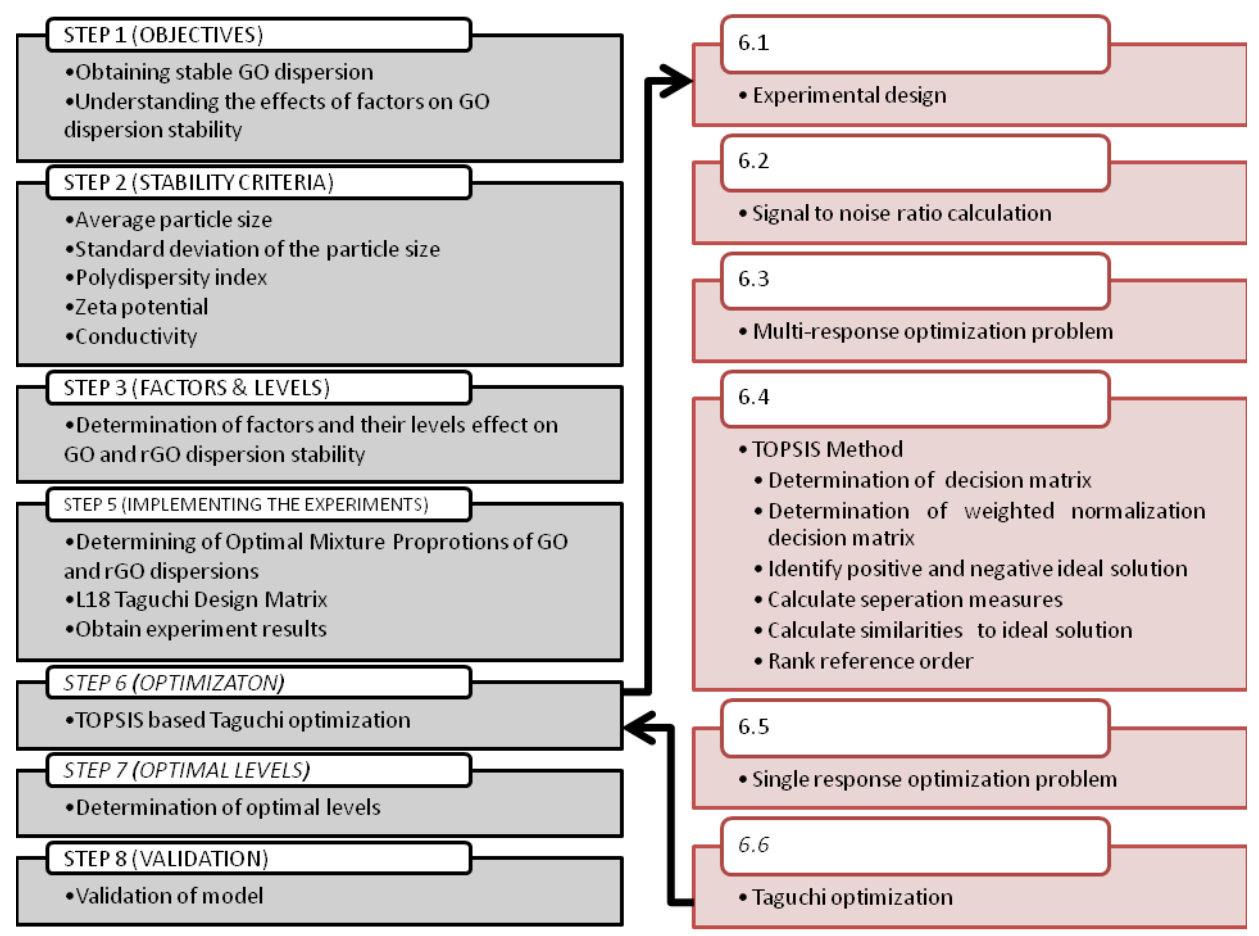

Fig. 1 Proposed methodology 
Table 1 Quality characteristic and their weights

\begin{tabular}{|c|c|c|c|c|c|c|c|}
\hline $\begin{array}{l}\text { Quality } \\
\text { Criteria }\end{array}$ & Symbol & Description & Information & $\begin{array}{l}\text { Target values } \\
\text { for GO }\end{array}$ & $\begin{array}{l}\text { Target values } \\
\text { for rGO }\end{array}$ & Weights & $\begin{array}{l}\text { Normalized } \\
\text { weights }\end{array}$ \\
\hline 1 & APS & Average particle size (nm) & Industrial usage & Smaller is better & Smaller is better & 1 & 0.20 \\
\hline 2 & SPS & $\begin{array}{l}\text { Standard deviation } \\
\text { of the particle size }(\mathrm{nm})\end{array}$ & Stability improvement & Smaller is better & Smaller is better & 1 & 0.20 \\
\hline 3 & PDI & Average polydispersity index & Monodispersity criterion & Smaller is better & Smaller is better & 1 & 0.20 \\
\hline 4 & $\mathrm{ZP}$ & Zeta-potential values $(\mathrm{mV})$ & Dispersion stability & Smaller is better & Smaller is better & 1 & 0.20 \\
\hline 5 & $\mathrm{CO}$ & Conductivity $(\mathrm{mS} / \mathrm{cm})$ & Industrial usage & Larger is better & Larger is better & 1 & 0.20 \\
\hline Total & & & & & & 4 & 1.0 \\
\hline
\end{tabular}

potential value and conductivity. The factors and their levels have been determined in consideration with the preliminary test results and usage rates in the literature [2, 32].

The TOPSIS-based Taguchi method has been used to improve the stability of both graphene oxide and reduced graphene oxide dispersions. Lastly; the improvement rate of the GO and rGO dispersion properties has been calculated and these results have been compared with the other studies in the literature.

\section{Identifying Conditions of Dispersions 4.1 Determination criteria and constraints of GO and rGO dispersions}

Dispersion properties of GO and rGO have been selected as the average particle size, standard deviation of the particle size, polydispersity index, zeta potential and conductivity value. For the GO and rGO dispersions; the smaller average particle size and standard deviation of the particle size have been selected as target value to use in the ink jet printing, super capacitor [41] and lithium ion battery technology [42]. Polydispersity index is requires to be minimized and provides an information that this dispersion is monodisperse or not [43]. Zeta potential values which should be minimized are critical parameter that shows the dispersion stability $[26,44]$. The dispersions with a zeta potential value of less than $-30 \mathrm{mV}$ are considered strongly as stable [45]. When the conductive ink production is considered, the conductivity value which should be maximized [46]. The factor levels that affect on the dispersion properties have been selected with regard to the preliminary test results and GO and rGO synthesis procedure in the literature [1-2, 32, 47-53].

\subsection{Determination of factors and their levels}

One factor that each has two control levels and seven factors that each has three control levels affects the GO and rGO stability. The factors' effect on GO dispersion stability has been selected as the graphite size (A), graphite (B), sodium nitrate amount (C), sulfuric acid (D), phosphoric acid (E), potassium permanganate amount $(\mathrm{F})$, oven temperature $(\mathrm{G})$ and mixing rate $(\mathrm{H})$ (Table 2). The factors' effect on rGO dispersion stability has been also selected as the drying process $\left(\mathrm{X}_{1}\right)$, reaction temperature $\left(\mathrm{X}_{2}\right)$, residence time in ultrasonic bath $\left(\mathrm{X}_{3}\right)$, reaction time $\left(\mathrm{X}_{4}\right)$, the amount of $\mathrm{GO}\left(\mathrm{X}_{5}\right)$, the amount of reducer $\left(\mathrm{X}_{6}\right)$, the type of reducer $\left(\mathrm{X}_{7}\right)$ and solution amount $\left(\mathrm{X}_{8}\right)$ (Table 2).

\subsection{Selecting the experimental design}

In this study a Taguchi orthogonal array $\left(\mathrm{L}_{18}\right)$ has been selected to record the experiment results. In Table 3, columns 2-9 and 10-17 represent the eight control factors and their uncoded levels for GO and rGO, respectively. This model has provided the eight performance measures simultaneously in order to analyze the factor effects.

In all experiments, average particle size which is calculated, standard deviation of the particle size, polydispersity index, zeta potential and conductivity value of GO and $\mathrm{rGO}$ dispersions have been transferred to Table 4 .

\section{Characterization of $\mathbf{G O}$ and rGO}

FTIR spectroscopy has been used to characterize the synthesized graphene oxide in Fig. 2. The absorption band at $3364 \mathrm{~cm}^{-1}$ is indicates that the presence of oxygen containing groups (O-H stretching vibrations) [54]. The absorption peak at $1714 \mathrm{~cm}^{-1}$ and $1618 \mathrm{~cm}^{-1}$ can be designated to $\mathrm{C}=\mathrm{O}$ stretching of carboxylic and/or carbonyl moiety functional groups [55]. The last absorption peaks at about $1222 \mathrm{~cm}^{-1}$, $1046 \mathrm{~cm}^{-1}$ and $579 \mathrm{~cm}^{-1}$ are designated to the C-O hydroxyl and epoxy groups stretching vibrations and the epoxy (C-O-C) stretching mode, respectively [2; 55]. FTIR depicts show that the synthesis of graphene oxide by Hummers method has been successfully done for the each experimental runs.

After the reducing agent addition, $\mathrm{H}_{2} \mathrm{O}$ molecules and much of the groups which contains oxide (Carbonyl $\mathrm{C}=\mathrm{O}$, hydroxyl $\mathrm{O}-\mathrm{H}$ ) of GO are removed with the regard to FTIR spectra (Fig. 3) [56].

The Raman spectrum of GO and rGO samples synthesized by Taguchi orthogonal arrays illustrates a G-band at $1590 \mathrm{~cm}^{-1}$, D band at $1350 \mathrm{~cm}^{-1}, 2 \mathrm{D}$ and $\mathrm{D}+\mathrm{D}^{\prime}$ band at $2700 \mathrm{~cm}^{-1}$ and $2930 \mathrm{~m}^{-1}$, respectively [57] (Fig. 4 and 5). The excitation wavelength and the excitation leaser energy have been selected as $780 \mathrm{~nm}$ and $10 \mathrm{mV}$, respectively. Raman results also demonstrate that the synthesis of graphene oxide by Hummers method has been succeeded for the each experimental runs (Fig. 4). 
Table 2 Factors and their levels

\begin{tabular}{|c|c|c|c|c|c|}
\hline & \multirow{2}{*}{ Symbol } & \multirow{2}{*}{ Factor } & \multicolumn{3}{|c|}{ Levels } \\
\hline & & & 1 & 2 & 3 \\
\hline \multirow{8}{*}{ GO } & $\mathrm{A}$ & Graphite size, $<\mu \mathrm{m}$ & 20 & 50 & $\mathrm{~N} / \mathrm{A}$ \\
\hline & B & Graphite amount, $g$ & 2.5 & 5.0 & 7.5 \\
\hline & $\mathrm{C}$ & $\mathrm{NaNO}_{3}$ amount (g) & 0 & 5 & 10 \\
\hline & $\mathrm{D}$ & $\mathrm{H}_{2} \mathrm{SO}_{4}$ amount $(\mathrm{ml})$ & 57.5 & 115.0 & 172.5 \\
\hline & $\mathrm{E}$ & $\mathrm{H}_{3} \mathrm{PO}_{4}$ amount $(\mathrm{ml})$ & 5.75 & 11.5 & 17.25 \\
\hline & $\mathrm{F}$ & $\mathrm{KMnO}_{4}$ amount $(\mathrm{g})$ & 7.5 & 15.0 & 22.5 \\
\hline & G & Oven temperature, ${ }^{\circ} \mathrm{C}$ & 40 & 60 & 80 \\
\hline & $\mathrm{H}$ & Mixing rate, rpm & 200 & 400 & 600 \\
\hline \multirow{8}{*}{ rGO } & $\mathrm{X}_{1}$ & Drying process at $50^{\circ} \mathrm{C}$ & Vacuumed & Non-vacuumed & N/A \\
\hline & $\mathrm{X}_{2}$ & Reaction temperature, ${ }^{\circ} \mathrm{C}$ & 70 & 95 & 120 \\
\hline & $\mathrm{X}_{3}$ & $\begin{array}{l}\text { Residence time in } \\
\text { ultrasonic bath, } h\end{array}$ & 2 & 4 & 6 \\
\hline & $\mathrm{X}_{4}$ & Reaction time, $\mathrm{h}$ & 8 & 24 & 48 \\
\hline & $\mathrm{X}_{5}$ & Graphene oxide amount, $\mathrm{g}$ & 1 & 2 & 3 \\
\hline & $\mathrm{X}_{6}$ & The amount of reducer, $g$ & 3 & 6 & 9 \\
\hline & $\mathrm{X}_{7}$ & The type of reducer & $\mathrm{NaHB}_{4}$ & D-fructose & Ascorbic acid \\
\hline & $\mathrm{X}_{8}$ & Solution amount, $\mathrm{ml}$ & 200 & 400 & 600 \\
\hline
\end{tabular}

Table $3 \mathrm{~L}_{18}$ Taguchi design for GO and rGO dispersions

\begin{tabular}{|c|c|c|c|c|c|c|c|c|c|c|c|c|c|c|c|c|}
\hline \multirow{3}{*}{ No. } & \multicolumn{8}{|c|}{ GO } & \multicolumn{8}{|c|}{ rGO } \\
\hline & \multicolumn{8}{|c|}{ Uncoded levels } & \multicolumn{8}{|c|}{ Uncoded levels } \\
\hline & A & $\mathrm{B}$ & $\mathrm{C}$ & $\mathrm{D}$ & E & $\mathrm{F}$ & G & $\mathrm{H}$ & $X_{1}$ & $\mathrm{X}_{2}$ & $\mathrm{X}_{3}$ & $\mathbf{X}_{4}$ & $X_{5}$ & $X_{6}$ & $\mathbf{X}_{7}$ & $\mathbf{X}_{8}$ \\
\hline 1 & 20 & 2.5 & 0 & 57.5 & 5.75 & 7.5 & 40 & 200 & $\mathrm{~V}^{*}$ & 70 & 2 & 8 & 1 & 3 & $\mathrm{NaBH}_{4}$ & 200 \\
\hline 2 & 20 & 2.5 & 5 & 115.0 & 11.50 & 15 & 60 & 400 & V & 70 & 4 & 24 & 2 & 6 & D-fructose & 400 \\
\hline 3 & 20 & 2.5 & 10 & 172.5 & 17.25 & 22.5 & 80 & 600 & V & 70 & 6 & 48 & 3 & 9 & Ascorbic acid & 600 \\
\hline 4 & 20 & 5.0 & 0 & 57.5 & 11.50 & 15 & 80 & 600 & $\mathrm{~V}$ & 95 & 2 & 8 & 2 & 6 & Ascorbic acid & 600 \\
\hline 5 & 20 & 5.0 & 5 & 115.0 & 17.25 & 22.5 & 40 & 200 & $\mathrm{~V}$ & 95 & 4 & 24 & 3 & 9 & $\mathrm{NaBH}_{4}$ & 200 \\
\hline 6 & 20 & 5.0 & 10 & 172.5 & 5.75 & 7.5 & 60 & 400 & V & 95 & 6 & 48 & 1 & 3 & D-fructose & 400 \\
\hline 7 & 20 & 7.5 & 0 & 115.0 & 5.75 & 22.5 & 60 & 600 & V & 120 & 2 & 24 & 1 & 9 & D-fructose & 600 \\
\hline 8 & 20 & 7.5 & 5 & 172.5 & 11.50 & 7.5 & 80 & 200 & $\mathrm{~V}$ & 120 & 4 & 48 & 2 & 3 & Ascorbic acid & 200 \\
\hline 9 & 20 & 7.5 & 10 & 57.5 & 17.25 & 15 & 40 & 400 & V & 120 & 6 & 8 & 3 & 6 & $\mathrm{NaBH}_{4}$ & 400 \\
\hline 10 & 50 & 2.5 & 0 & 172.5 & 17.25 & 15 & 60 & 200 & $\mathrm{NV}^{* *}$ & 70 & 2 & 48 & 3 & 6 & D-fructose & 200 \\
\hline 11 & 50 & 2.5 & 5 & 57.5 & 5.75 & 22.5 & 80 & 400 & NV & 70 & 4 & 8 & 1 & 9 & Ascorbic acid & 400 \\
\hline 12 & 50 & 2.5 & 10 & 115.0 & 11.50 & 7.5 & 40 & 600 & NV & 70 & 6 & 24 & 2 & 3 & $\mathrm{NaBH}_{4}$ & 600 \\
\hline 13 & 50 & 5.0 & 0 & 115.0 & 17.25 & 7.5 & 80 & 400 & NV & 95 & 2 & 24 & 3 & 3 & Ascorbic acid & 400 \\
\hline 14 & 50 & 5.0 & 5 & 172.5 & 5.75 & 15 & 40 & 600 & NV & 95 & 4 & 48 & 1 & 6 & $\mathrm{NaBH}_{4}$ & 600 \\
\hline 15 & 50 & 5.0 & 10 & 57.5 & 11.50 & 22.5 & 60 & 200 & NV & 95 & 6 & 8 & 2 & 9 & D-fructose & 200 \\
\hline 16 & 50 & 7.5 & 0 & 172.5 & 11.50 & 22.5 & 40 & 400 & NV & 120 & 2 & 48 & 2 & 9 & $\mathrm{NaBH}_{4}$ & 400 \\
\hline 17 & 50 & 7.5 & 5 & 57.5 & 17.25 & 7.5 & 60 & 600 & NV & 120 & 4 & 8 & 3 & 3 & D-fructose & 600 \\
\hline 18 & 50 & 7.5 & 10 & 115.0 & 5.75 & 15 & 80 & 200 & NV & 120 & 6 & 24 & 1 & 6 & Ascorbic acid & 200 \\
\hline
\end{tabular}


Table 4 Experimental results

\begin{tabular}{llllllllllll}
\hline $\begin{array}{l}\text { Exp. } \\
\text { No. }\end{array}$ & APS & SPS & PDI & ZP & CO & $\begin{array}{l}\text { Exp. } \\
\text { No. }\end{array}$ & APS & SPS & PDI & ZP & CO \\
\hline GO1 & 997.2 & 262.9 & 0.72 & -27.93 & 0.01 & rGO1 & 903.2 & 37.0 & 0.40 & -37.90 & 0.0015 \\
GO2 & 1753.0 & 265.0 & 0.45 & -35.83 & 0.01 & rGO2 & 456.9 & 10.7 & 0.44 & -26.73 & 0.0004 \\
GO3 & 309.6 & 51.6 & 0.43 & -36.70 & 0.07 & rGO3 & 496.5 & 41.2 & 0.53 & -27.70 & 0.0008 \\
GO4 & 1314.7 & 6.8 & 0.60 & -26.77 & 0.15 & rGO4 & 419.2 & 15.6 & 0.45 & -40.20 & 0.0005 \\
GO5 & 578.5 & 14.8 & 0.12 & -41.80 & 0.02 & rGO5 & 775.8 & 35.3 & 0.66 & -1.90 & 0.4710 \\
\hline GO6 & 2106.0 & 292.3 & 0.82 & -39.73 & 0.01 & rGO6 & 517.2 & 7.5 & 0.42 & -41.67 & 0.0002 \\
GO7 & 2391.0 & 244.6 & 0.52 & -23.70 & 0.18 & rGO7 & 655.1 & 41.0 & 0.56 & -24.20 & 0.0004 \\
GO8 & 2030.0 & 157.7 & 0.95 & -41.43 & 0.02 & rGO8 & 1378.0 & 33.1 & 0.57 & -24.87 & 0.0019 \\
GO9 & 2919.7 & 338.2 & 1.00 & -30.27 & 0.04 & rGO9 & 1245.0 & 96.9 & 0.66 & -33.83 & 0.0040 \\
GO10 & 538.6 & 17.6 & 0.56 & -30.87 & 0.05 & rGO10 & 525.4 & 24.3 & 0.53 & -42.70 & 0.0003 \\
\hline GO11 & 595.9 & 14.7 & 0.73 & -23.37 & 0.10 & rGO11 & 524.1 & 11.0 & 0.53 & -35.90 & 0.0006 \\
GO12 & 849.9 & 80.9 & 0.68 & -28.97 & 0.06 & rGO12 & 721.5 & 23.7 & 0.47 & -41.27 & 0.0008 \\
GO13 & 571.0 & 43.1 & 0.52 & -42.83 & 0.04 & rGO13 & 1025.1 & 50.9 & 0.55 & -41.63 & 0.0003 \\
GO14 & 1387.7 & 216.9 & 0.90 & -33.53 & 0.03 & rGO14 & 633.9 & 24.9 & 0.52 & -35.97 & 0.0020 \\
GO15 & 1057.9 & 97.0 & 1.00 & -31.20 & 0.02 & rGO15 & 686.9 & 42.5 & 0.53 & -37.00 & 0.0005 \\
\hline GO16 & 1016.8 & 68.1 & 0.77 & -31.67 & 0.01 & rGO16 & 1003.4 & 25.3 & 0.56 & -1.93 & 0.0345 \\
GO17 & 620.0 & 44.9 & 0.56 & -39.43 & 0.05 & rGO17 & 621.3 & 39.0 & 0.53 & -42.67 & 0.0004 \\
GO18 & 743.9 & 64.7 & 0.67 & -39.70 & 0.01 & rGO18 & 422.7 & 25.6 & 0.50 & -39.47 & 0.0005 \\
\hline
\end{tabular}

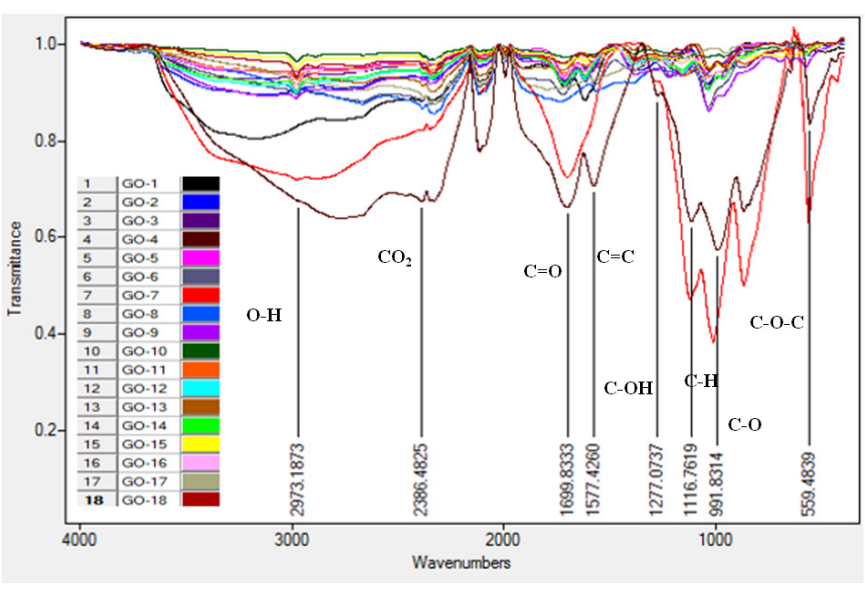

Fig. 2 FTIR spectra of GO samples prepared by $\mathrm{L}_{18}$ Taguchi design

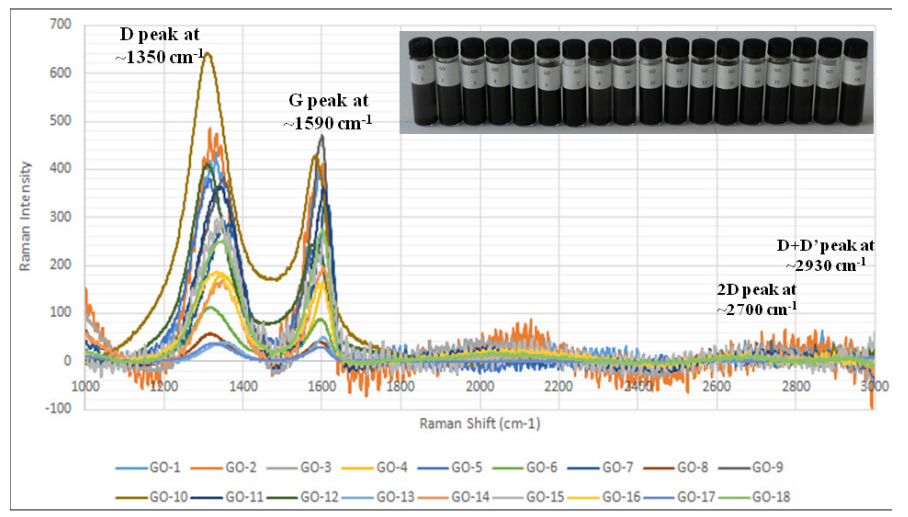

Fig. 4 Raman spectra of GO samples prepared by $\mathrm{L}_{18}$ Taguchi design

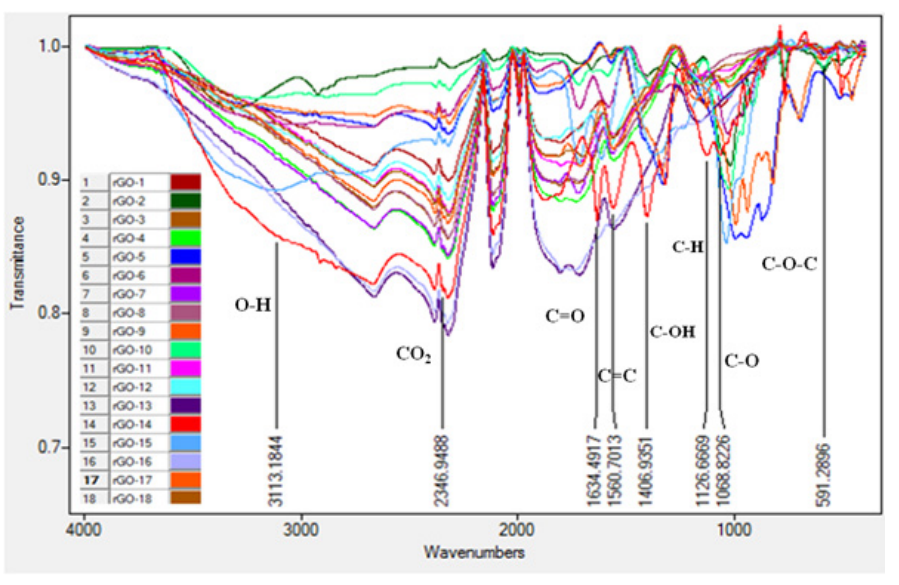

Fig. 3 FTIR spectra of rGO samples prepared by $\mathrm{L}_{18}$ Taguchi design

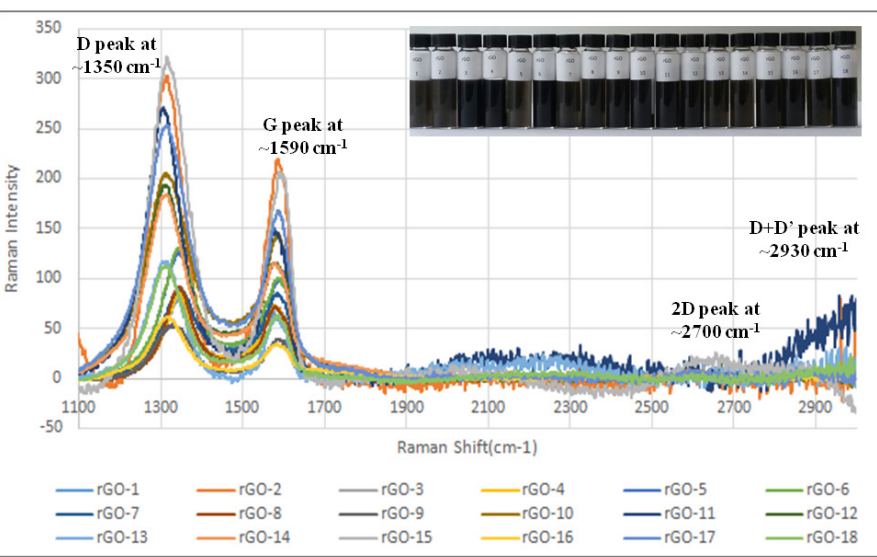

Fig. 5 Raman spectra of rGO samples prepared by $\mathrm{L}_{18}$ Taguchi design 
As it is expected, ID/IG values of GO are larger than the ID/ IG values of rGO as a result of reduction process by means of the formation of some new and smaller $\mathrm{sp}^{2}$ domains [58-59].

\section{Optimization}

\subsection{TOPSIS based Taguchi Optimization of the synthesized graphene oxide}

TOPSIS based Taguchi method provides the five performance measures (responses) simultaneously in order to solve the multi-response-optimization problem. The $\mathrm{S} / \mathrm{N}$ ratios, as the smaller and larger is better, are calculated with the use of Eq. (1) and (2) for each response. The experimental design and the $\mathrm{S} / \mathrm{N}$ ratios as the decision matrix are given in Table 5, columns 2-6. The TOPSIS method converts the multi-response optimization problem into a single response problem [60]. The final results are illustrated in Table 5, last column [61].

The normalization method is one of the last stages of the TOPSIS method that led to the final optimal design of GO5 (Table 5) through the surrogate responses, $\mathrm{C}_{\mathrm{i}}{ }^{*}$.

\subsection{Optimum graphene oxide}

The quality contributions of $\mathrm{S} / \mathrm{N}$ ratios for these five responses have been predicted with the use of an optimal mixture level to predict the improvement rate under the selected optimum conditions between the estimated mixture levels. The significant improvement rate which is obtained by TOPSISTaguchi method is given in Table 6 .

\subsection{TOPSIS based Taguchi Optimization of the synthesized reduced graphene oxide}

TOPSIS based Taguchi method provides the five performance measures (responses) simultaneously in order to solve the multi-response-optimization problem (in Table 7, columns 2-6). The $\mathrm{S} / \mathrm{N}$ ratios, for the smaller and larger is better, are calculated with the use of Eq. (1) and (2) for each response. The weighted decision matrix is given in Table 7 , columns 7-11. The positive ideal solution $\left(A^{*}\right)$, the negative ideal solution $\left(A^{-}\right)$and the similarity of the ideal solutions in each scenario, $\mathrm{Sc},\left(\mathrm{C}_{\mathrm{i}}^{*}\right)$ have been given in Table 7 [61].

Table $5 \mathrm{~S} / \mathrm{N}$ ratios calculated by Minitab ${ }^{\circledR}$, and TOPSIS method implementation for GO dispersions

\begin{tabular}{|c|c|c|c|c|c|c|c|c|c|c|c|c|c|}
\hline \multirow[b]{2}{*}{ Response } & \multicolumn{4}{|c|}{ Decision Matrix (S/N Ratios) } & \multicolumn{6}{|c|}{ Weighted Normalized Decision Matrix } & \multirow[t]{3}{*}{$\mathbf{S}_{\mathbf{i}}^{*}$} & \multirow[t]{3}{*}{$\mathbf{S}_{\mathbf{i}}^{-}$} & \multirow[t]{3}{*}{$\mathbf{C}_{\mathbf{i}}^{*}$} \\
\hline & APS & SPS & PDI & $\mathbf{Z P}$ & $\mathrm{CO}$ & \multirow[b]{2}{*}{$v_{i l}$} & \multirow[b]{2}{*}{$v_{i 2}$} & \multirow[b]{2}{*}{$v_{i 3}$} & \multirow[b]{2}{*}{$\mathrm{v}_{\mathrm{i} 4}$} & \multirow[b]{2}{*}{$\mathrm{v}_{\mathrm{i} 5}$} & & & \\
\hline Weight & $0.200^{b}$ & 0.200 & 0.200 & 0.200 & 0.200 & & & & & & & & \\
\hline GO1 & -59.98 & -48.40 & 2.85 & 28.92 & -40.00 & -0.05 & -0.06 & 0.02 & 0.04 & -0.06 & 0.14 & 0.02 & 0.15 \\
\hline GO2 & -64.88 & -48.46 & 6.94 & 31.08 & -40.00 & -0.05 & -0.06 & 0.06 & 0.05 & -0.06 & 0.11 & 0.06 & 0.34 \\
\hline GO3 & -49.82 & -34.25 & 7.33 & 31.29 & -23.10 & -0.04 & -0.04 & 0.06 & 0.05 & -0.04 & 0.09 & 0.07 & 0.43 \\
\hline GO4 & -62.38 & -16.65 & 4.44 & 28.55 & -16.48 & -0.05 & -0.02 & 0.04 & 0.04 & -0.03 & 0.11 & 0.07 & 0.37 \\
\hline${ }^{\circ}$ GO5 & $-55.25^{b}$ & -23.41 & 18.42 & 32.42 & -33.98 & $-0.04^{b}$ & -0.03 & 0.15 & 0.05 & -0.05 & $0.03^{c}$ & $0.15^{d}$ & $0.83^{\mathrm{e}}$ \\
\hline GO6 & -66.47 & -49.32 & 1.72 & 31.98 & -40.00 & -0.05 & -0.06 & 0.01 & 0.05 & -0.06 & 0.15 & 0.02 & 0.10 \\
\hline GO7 & -67.57 & -47.77 & 5.68 & 27.49 & -14.89 & -0.05 & -0.06 & 0.05 & 0.04 & -0.02 & 0.11 & 0.06 & 0.35 \\
\hline GO8 & -66.15 & -43.96 & 0.45 & 32.35 & -33.98 & -0.05 & -0.05 & 0.00 & 0.05 & -0.05 & 0.15 & 0.02 & 0.09 \\
\hline GO9 & -69.31 & -50.58 & 0.00 & 29.62 & -27.96 & -0.05 & -0.06 & 0.00 & 0.05 & -0.04 & 0.16 & 0.02 & 0.11 \\
\hline GO10 & -54.63 & -24.91 & 5.04 & 29.79 & -26.02 & -0.04 & -0.03 & 0.04 & 0.05 & -0.04 & 0.11 & 0.06 & 0.34 \\
\hline GO11 & -55.50 & -23.35 & 2.73 & 27.37 & -20.00 & -0.04 & -0.03 & 0.02 & 0.04 & -0.03 & 0.13 & 0.05 & 0.29 \\
\hline GO12 & -58.59 & -38.16 & 3.35 & 29.24 & -24.44 & -0.05 & -0.05 & 0.03 & 0.05 & -0.04 & 0.12 & 0.04 & 0.24 \\
\hline GO13 & -55.13 & -32.69 & 5.68 & 32.63 & -27.96 & -0.04 & -0.04 & 0.05 & 0.05 & -0.04 & 0.11 & 0.06 & 0.34 \\
\hline GO14 & -62.85 & -46.73 & 0.92 & 30.51 & -30.46 & -0.05 & -0.06 & 0.01 & 0.05 & -0.05 & 0.15 & 0.02 & 0.11 \\
\hline GO15 & -60.49 & -39.74 & 0.00 & 29.88 & -33.98 & -0.05 & -0.05 & 0.00 & 0.05 & -0.05 & 0.15 & 0.02 & 0.10 \\
\hline G016 & -60.14 & -36.66 & 2.27 & 30.01 & -40.00 & -0.05 & -0.04 & 0.02 & 0.05 & -0.06 & 0.14 & 0.03 & 0.16 \\
\hline GO17 & -55.85 & -33.04 & 5.04 & 31.92 & -26.02 & -0.04 & -0.04 & 0.04 & 0.05 & -0.04 & 0.11 & 0.05 & 0.32 \\
\hline \multirow[t]{4}{*}{ G018 } & -57.43 & -36.22 & 3.48 & 31.98 & -40.00 & -0.04 & -0.04 & 0.03 & 0.05 & -0.06 & 0.13 & 0.03 & 0.21 \\
\hline & $256.07^{a}$ & 164.6 & 24.98 & 129.13 & 131.7 & & & & & & & & \\
\hline & & & & & $A^{*}=$ & -0.04 & -0.02 & 0.15 & 0.05 & -0.02 & & & \\
\hline & & & & & $A-=$ & -0.05 & -0.06 & 0.00 & 0.04 & -0.06 & & & \\
\hline
\end{tabular}

${ }^{a}$ The square root of sum of squares of each element in the columns

${ }^{\mathrm{b}}$ From $[31]: 0.200 *[(-55.25) /(256.07)]=\mathbf{- 0 . 0 4}$;

${ }^{\mathrm{c}}$ From $[31]:\left\{[(-0.04)-(-0.04)]^{2}+\ldots . .+[-(0.05)-(-0.02)]^{2}\right\}^{1 / 2}=\mathbf{0 . 0 3}$

${ }^{\mathrm{d}}$ From[31]: $\left\{[(-0.04)-(-0.05)]^{2} \ldots \ldots . .+[-(0.05)-(-0.06)]^{2}\right\}^{1 / 2}=\mathbf{0 . 1 5}$

${ }^{\mathrm{e}}$ From $[31]: 0.03 /(0.03+0.15)=\mathbf{0 . 8 3}$

${ }^{\circ} \mathrm{GO} 5$ : Optimum experiment 
Table 6 Improvement ratio between optimum and reference GO dispersion

\begin{tabular}{lllll}
\hline Responses & Definition & ${ }^{\mathbf{0}} \mathbf{G O 6}$ & ${ }^{*} \mathbf{G O 5}$ & Improvement rate $^{\mathbf{1}}(\mathbf{\%})$ \\
\hline $\mathbf{1}$ & Average particle size (nm) & 2106.0 & 578.5 & 264.0 \\
$\mathbf{2}$ & Standard deviation of the particle size (nm) & 292.3 & 14.8 & 1875.0 \\
$\mathbf{3}$ & Average polydispersity index & 0.82 & 0.12 & 583.3 \\
$\mathbf{4}$ & Zeta-potential values (mV) & -39.73 & -41.80 & 5.0 \\
$\mathbf{5}$ & Conductivity (mS/cm) & 0.01 & 0.02 & 50.0 \\
\hline
\end{tabular}

${ }^{\circ}$ Predicted levels before the experimental design (reference GO dispersion)

"Experiment results with the highest grading score (optimum GO dispersion)

$1\left[\left(\frac{2106.0-578.5}{578.5}\right) * 100\right]=264.0$

Table $7 \mathrm{~S} / \mathrm{N}$ ratios calculated by Minitab ${ }^{\circledR}$, and TOPSIS method implementation for rGO dispersions

\begin{tabular}{|c|c|c|c|c|c|c|c|c|c|c|c|c|c|}
\hline \multicolumn{6}{|c|}{ Decision Matrix (S/N Ratios) } & \multicolumn{5}{|c|}{ Weighted Normalized Decision Matrix } & \multirow[t]{3}{*}{$S_{i}^{*}$} & \multirow[t]{3}{*}{$\mathrm{S}_{\mathrm{i}}^{-}$} & \multirow[t]{3}{*}{$\mathrm{C}_{\mathrm{i}}^{*}$} \\
\hline Response & APS & SPS & PDI & $\mathbf{Z P}$ & $\mathrm{CO}$ & \multirow{2}{*}{$v_{i I}$} & \multirow{2}{*}{$v_{i 2}$} & \multirow{2}{*}{$v_{i 3}$} & \multirow[b]{2}{*}{$v_{i 4}$} & \multirow[b]{2}{*}{$v_{i 5}$} & & & \\
\hline Weight & $0.200^{b}$ & 0.200 & 0.200 & 0.200 & 0.200 & & & & & & & & \\
\hline rGO1 & -59.12 & -31.36 & 7.96 & 31.57 & -56.48 & -0.05 & -0.05 & 0.06 & 0.05 & -0.04 & 0.05 & 0.06 & 0.55 \\
\hline rGO2 & -53.20 & -20.59 & 7.13 & 28.54 & -67.96 & -0.04 & -0.03 & 0.06 & 0.05 & -0.05 & 0.05 & 0.06 & 0.54 \\
\hline rGO3 & -53.92 & -32.30 & 5.51 & 28.85 & -61.94 & -0.04 & -0.05 & 0.04 & 0.05 & -0.05 & 0.05 & 0.04 & 0.45 \\
\hline rGO4 & -52.45 & -23.86 & 6.94 & 32.08 & -66.02 & -0.04 & -0.04 & 0.06 & 0.05 & -0.05 & 0.05 & 0.06 & 0.55 \\
\hline rGO5 & -57.79 & -30.96 & 3.61 & 5.58 & -6.54 & -0.05 & -0.05 & 0.03 & 0.01 & -0.01 & 0.06 & 0.05 & 0.47 \\
\hline${ }^{\circ}$ rGO6 & $-54.27^{b}$ & -17.50 & 7.54 & 32.40 & -73.98 & $-0.05^{b}$ & -0.03 & 0.06 & 0.05 & -0.06 & $0.05^{c}$ & $0.06^{d}$ & $0.55^{\mathrm{e}}$ \\
\hline rGO7 & -56.33 & -32.26 & 5.04 & 27.68 & -67.96 & -0.05 & -0.05 & 0.04 & 0.04 & -0.05 & 0.06 & 0.04 & 0.40 \\
\hline rGO8 & -62.78 & -30.40 & 4.88 & 27.91 & -54.42 & -0.05 & -0.05 & 0.04 & 0.04 & -0.04 & 0.05 & 0.04 & 0.46 \\
\hline rGO9 & -61.90 & -39.73 & 3.61 & 30.59 & -47.96 & -0.05 & -0.06 & 0.03 & 0.05 & -0.04 & 0.06 & 0.05 & 0.43 \\
\hline rGO10 & -54.41 & -27.71 & 5.51 & 32.61 & -70.46 & -0.05 & -0.04 & 0.04 & 0.05 & -0.05 & 0.06 & 0.05 & 0.48 \\
\hline rGO11 & -54.39 & -20.83 & 5.51 & 31.10 & -64.44 & -0.05 & -0.03 & 0.04 & 0.05 & -0.05 & 0.05 & 0.05 & 0.52 \\
\hline rGO12 & -57.16 & -27.49 & 6.56 & 32.31 & -61.94 & -0.05 & -0.04 & 0.05 & 0.05 & -0.05 & 0.05 & 0.05 & 0.53 \\
\hline rG013 & -60.22 & -34.13 & 5.19 & 32.39 & -70.46 & -0.05 & -0.06 & 0.04 & 0.05 & -0.05 & 0.06 & 0.05 & 0.43 \\
\hline rGO14 & -56.04 & -27.92 & 5.68 & 31.12 & -53.98 & -0.05 & -0.05 & 0.05 & 0.05 & -0.04 & 0.04 & 0.05 & 0.53 \\
\hline rG015 & -56.74 & -32.57 & 5.51 & 31.36 & -66.02 & -0.05 & -0.05 & 0.04 & 0.05 & -0.05 & 0.06 & 0.05 & 0.45 \\
\hline rG016 & -60.03 & -28.06 & 5.04 & 5.71 & -29.24 & -0.05 & -0.05 & 0.04 & 0.01 & -0.02 & 0.06 & 0.04 & 0.43 \\
\hline rGO17 & -55.87 & -31.82 & 5.51 & 32.60 & -67.96 & -0.05 & -0.05 & 0.04 & 0.05 & -0.05 & 0.06 & 0.05 & 0.46 \\
\hline \multirow[t]{4}{*}{ rGO18 } & -52.52 & -28.16 & 6.02 & 31.93 & -66.02 & -0.04 & -0.05 & 0.05 & 0.05 & -0.05 & 0.05 & 0.05 & 0.50 \\
\hline & $240.6^{\mathrm{a}}$ & 124.03 & 24.70 & 124.20 & 257.72 & & & & & & & & \\
\hline & & & & & $A^{*}=$ & -0.04 & -0.03 & 0.06 & 0.05 & -0.01 & & & \\
\hline & & & & & $A-=$ & -0.05 & -0.06 & 0.03 & 0.01 & -0.06 & & & \\
\hline
\end{tabular}

aThe square root of sum of squares of each element in the columns

${ }^{\mathrm{b}}$ From [31] : 0.200*[(-54.27)/(240.6)] $=-0.05$;

'From[31]: $\left\{[(-0.05)-(-0.04)]^{2}+\ldots \ldots+[-(0.06)-(-0.01)]^{2}\right\}^{1 / 2}=0.05$

${ }^{\mathrm{d}}$ From $[31]:\left\{[(-0.05)-(-0.05)]^{2} \ldots \ldots . .+[-(0.06)-(-0.06)]^{2}\right\}^{1 / 2}=0.06$

eFrom[31] : 0.06/(0.06+0.05) $=0.55$

${ }^{\circ}$ rGO6: Optimum experiment 
The normalization methods have led to the final optimum design of rGO6 (Table 7) with the use of the surrogate responses, $\mathrm{C}_{\mathrm{i}}^{*}$.

\subsection{Optimum rGO}

The quality contributions of $\mathrm{S} / \mathrm{N}$ ratios for five of eight responses have been predicted with the use of an optimal mixture level to predict the improvement rate under the selected optimum conditions between the estimated mixture levels. The significant improvement rate which is obtained by TOPSISTaguchi method is given in Table 8 .

\section{Discussion}

\subsection{Analysis of the factor effects}

When the results of regression analysis (Table 9) are evaluated, it can be seen that the smaller graphite size causes the smaller graphene oxide particle size (with the $0.027 \mathrm{p}$-value). The average particle size has increased with the increasing amount of graphite. The variability in the product has been decreased with the increasing amount of $\mathrm{H}_{3} \mathrm{PO}_{4}$. The regression analysis results show that higher graphite size causes less product variance, with the $0.030 \mathrm{p}$-value. The zeta potential of graphene oxide dispersions has decreased with the increasing amount of $\mathrm{H}_{3} \mathrm{PO}_{4}$ and $\mathrm{H}_{2} \mathrm{SO}_{4}$, with the 0.077 and 0.084 p-values, respectively. The conductivity of graphene oxide samples has been decreased with the usage of sodium nitrate and increased significantly with the high mixture rate, with the 0.008 p-values. The amount of reducer causes a significantly higher variance on zeta potential and polydispersity index, with the 0.037 and 0.012 p-values. The polydispersity index of rGO dispersions has been increased with increasing amount of graphene oxide, with the $0.015 \mathrm{p}$-values. The low reaction temperature has been preferred in order to obtain graphene oxide samples which have the low polydispersity index. The standard deviation of the rGO dispersions is significantly influenced by the synergetic impact of graphene oxide amount.

\subsection{Statistical comparison}

The paired-t test has been used to compare classical Hummers method (GO14) and the improved Hummers method (GO4) in order to prepare the graphene oxide dispersions, statistically. Similarly, the paired-t test has been also used to determine whether there was a statistically significant difference in the preparing stable dispersion between the ascorbic acid and sodium borohydride as the most preferred chemical GO reducing agents [62]. There is no statistically significant difference has been found between the improved Hummers and the classical Hummers method and ascorbic acid and sodium borohydride on product stability (Table 10).

\subsection{The effectiveness of experimental design study}

The findings which have been obtained by this study have been compared with the other studies in the literature to determine the effectiveness of variance reduction study [30]. The studies related to the standard deviation of graphene oxide dispersions' particle size in the literature differ in the device that the measurement is done. For example, while the standard deviation of particle's size was obtained as $200 \mathrm{~nm}$ for the graphene oxide and $1180 \mathrm{~nm}$ for the reduced graphene oxide in the studies which were done with the use of Transition Electron Microscopy (TEM) or Scanning Electron Microscopy (SEM) [63-64] the values of standard deviation which were obtained with the acoustic spectrometer decreased to $10 \mathrm{~nm}$ [22]. Thus, the values which were obtained in the study were compared with the measurements which were done with the particle size analyzer in order to determine the efficacy of optimization study. Obreja et al. [30] determined the standard deviation of particle size as $29.1 \mathrm{~nm}$ as the graphene oxide which was obtained with Hummers method in their study. While the optimum particle size which was obtained for the graphene oxide in this study was found as $14.8 \mathrm{~nm}$, it was found as $7.5 \mathrm{~nm}$ for the reduced graphene oxide. Moreover, when the reference experiment was obtained, the improvement ratio was found as $1875.0 \%$ for the

Table 8 Improvement ratio between optimum and reference rGO dispersion

\begin{tabular}{|c|c|c|c|c|}
\hline Responses & Definition & ${ }^{\circ}$ rGO1 & *rGO6 & Improvement rate ${ }^{1}(\%)$ \\
\hline 1 & Average particle size $(\mathrm{nm})$ & 903.2 & 517.2 & 42.7 \\
\hline 2 & Standard deviation of the particle size $(\mathrm{nm})$ & 37.0 & 7.5 & 79.7 \\
\hline 3 & Average polydispersity index & 0.40 & 0.42 & -5.0 \\
\hline 4 & Zeta-potential values $(\mathrm{mV})$ & -37.90 & -41.67 & 9.9 \\
\hline 5 & Conductivity $(\mathrm{mS} / \mathrm{cm})$ & 0.0015 & 0.0002 & -86.7 \\
\hline \multicolumn{5}{|c|}{ Predicted levels before the experimental design (reference rGO dispersion) } \\
\hline \multicolumn{5}{|c|}{ Experiment results with the highest grading score (optimum rGO dispersion) } \\
\hline${ }^{1}\left[\left(\frac{903.2-517.2}{517.2}\right)\right.$ & $* 100]=42.7$ & & & \\
\hline
\end{tabular}


Table 9 Factor effects and associated p-values of GO and rGO dispersions

\begin{tabular}{|c|c|c|c|c|c|c|c|c|c|c|c|}
\hline & \multirow{3}{*}{$\begin{array}{l}\text { Source } \\
\text { (Factors) }\end{array}$} & \multicolumn{10}{|c|}{ Responses } \\
\hline & & \multicolumn{2}{|c|}{ APS (nm) } & \multicolumn{2}{|c|}{ SPS (nm) } & \multicolumn{2}{|l|}{ PDI } & \multicolumn{2}{|c|}{$\mathrm{ZP}(\mathrm{mV})$} & \multicolumn{2}{|c|}{$\mathrm{CO}(\mathrm{S} / \mathrm{m})$} \\
\hline & & T-value & p-value & T-value & p-value & T-value & $\mathrm{p}$-value & T-value & $\mathrm{p}$-value & T-value & p-value \\
\hline \multirow{8}{*}{ GO } & Graphite size, $<\mu \mathrm{m}$ & -2.65 & $0.027 *$ & -2.58 & $0.030 *$ & 0.76 & 0.468 & 0.12 & 0.906 & -0.95 & 0.367 \\
\hline & Graphite amount, g & 2.16 & $0.059 *$ & 0.72 & 0.488 & 1.07 & 0.312 & -1.29 & 0.230 & 0.08 & 0.936 \\
\hline & NaNO3 amount (g) & 0.54 & 0.606 & 0.90 & 0.390 & 1.08 & 0.307 & -1.30 & 0.225 & -1.91 & $0.088 *$ \\
\hline & H2SO4 amount (ml) & -0.05 & 0.958 & 0.13 & 0.901 & -0.21 & 0.835 & -2.00 & $0.077 *$ & -1.50 & 0.169 \\
\hline & H3PO4 amount (ml) & -1.24 & 0.247 & -1.88 & $0.093 *$ & -1.39 & 0.198 & -1.94 & $0.084 *$ & -0.58 & 0.574 \\
\hline & KMnO4 amount (g) & -0.57 & 0.585 & -1.25 & 0.241 & -0.81 & 0.439 & 1.82 & 0.102 & 1.75 & 0.115 \\
\hline & Oven temperature, ${ }^{\circ} \mathrm{C}$ & -1.01 & 0.339 & -2.06 & $0.069 *$ & -0.35 & 0.798 & -0.95 & 0.366 & 1.83 & 0.101 \\
\hline & Mixing rate, rpm & 0.43 & 0.679 & 0.10 & 0.923 & -0.39 & 0.703 & 1.36 & 0.206 & 3.41 & $0.008 *$ \\
\hline \multirow{7}{*}{ rGO } & Drying process at $50^{\circ} \mathrm{C}$ & -0.58 & 0.578 & -0.66 & 0.525 & 0.14 & 0.893 & -1.50 & 0.168 & -0.91 & 0.386 \\
\hline & Reaction temperature, ${ }^{\circ} \mathrm{C}$ & 1.76 & 0.112 & 1.79 & 0.107 & 2.72 & 0.024 & 1.40 & 0.196 & 0.09 & 0.927 \\
\hline & $\begin{array}{l}\text { Residence time in ultra- } \\
\text { sonic bath, } \mathrm{h}\end{array}$ & -0.46 & 0.658 & 0.69 & 0.510 & 0.34 & 0.741 & -1.00 & 0.344 & -0.08 & 0.940 \\
\hline & $\begin{array}{l}\text { Residence time in ultra- } \\
\text { sonic bath, h }\end{array}$ & 0.22 & 0.833 & -1.32 & 0.218 & 0.12 & 0.907 & 1.51 & 0.166 & -0.07 & 0.946 \\
\hline & Reaction time, $\mathrm{h}$ & 1.07 & 0.312 & 2.23 & $0.053 *$ & 3.01 & $0.015 *$ & 0.76 & 0.466 & 1.19 & 0.264 \\
\hline & The amount of reducer, $g$ & -1.06 & 0.316 & 0.08 & 0.937 & 2.44 & $0.037 *$ & 3.13 & $0.012 *$ & 1.27 & 0.236 \\
\hline & The type of reducer & -1.05 & 0.319 & -1.04 & 0.325 & -0.79 & 0.448 & -1.76 & 0.113 & -1.29 & 0.230 \\
\hline $\begin{array}{l}\text { Solution } \\
\text { amount, ml }\end{array}$ & Solution amount, $\mathrm{ml}$ & -1.19 & 0.266 & -0.20 & 0.849 & -0.74 & 0.480 & -0.87 & 0.407 & -1.19 & 0.264 \\
\hline
\end{tabular}

"significant at 10\% (p-value) has been shown in bold. $(+)$ Synergistic effect; (-) Antagonistic effect

Table 10.t-test results for statistical comparison

\begin{tabular}{|c|c|c|c|c|c|c|c|}
\hline \multirow[t]{2}{*}{ No. } & \multirow[t]{2}{*}{ Definition } & \multicolumn{4}{|c|}{ Number of experiments } & \multirow[t]{2}{*}{$\begin{array}{l}\text { T-test } \\
\text { Statistics }\end{array}$} & \multirow[t]{2}{*}{ p-value* } \\
\hline & & $\mathrm{GO}^{\mathrm{a}}$ & GO14 ${ }^{\beta}$ & $\mathrm{rGO}^{\mathrm{c}}$ & $\mathrm{rGO}^{\mathrm{d}}$ & & \\
\hline 1 & Average particle size (nm) & 1314.7 & 1387.7 & 903.2 & 419.2 & $-0.15^{1}$ & $0.888 \ddagger$ \\
\hline 2 & Standard deviation of the particle size $(\mathrm{nm})$ & 6.8 & 216.9 & 37.0 & 15.6 & $-0.51^{2}$ & $0.634^{\circ}$ \\
\hline 3 & Average polydispersity index & 0.6 & 0.90 & 0.40 & 0.45 & & \\
\hline 4 & Zeta-potential values (mV) & -26.77 & -33.53 & -37.90 & -40.20 & & \\
\hline 5 & Conductivity (mS/cm) & 0.15 & 0.03 & 0.0015 & 0.005 & & \\
\hline
\end{tabular}

graphene oxide nanofluid at the same concentration and $79.7 \%$ for the reduced graphene oxide nanofluid. If it is considered that the factor which affects mostly on the particle size is the graphene oxide concentration [23], these improvement ratios indicate that the mixture design in the production of stable dispersion is indispensable.

\section{Conclusions}

The subject on the stability of graphene oxide dispersions is very important to expand its usage fields. The graphene oxide in solution has a wide usage field from the drug industry to the multi-purpose $3 \mathrm{D}$ ink production. The following manners will increase the graphene oxide's usage ratio in the sectors that its stability properties are understood. Moreover, the carbon-based products will increase their superior properties better with the increase in stability.

In this study, the criteria that represent the stability of the graphene oxide and reduced graphene oxide dispersions are identified as the average particle size, standard deviation of the particle size, polydispersity index, zeta potential and conductivity. A TOPSIS based Taguchi optimization method have been used in this study to obtain the best possible mix proportions, which improve the stability, of the graphene oxide and reduced graphene oxide. The results show that the graphite size 
and the amount of reducer are the most influential factors on graphene oxide and reduced graphene oxide with the 0.027 and $0.012 \mathrm{p}$-values. The oxidants amount and reaction temperature have been also found as effective on the graphene oxide and reduced graphene oxide stability, respectively.

Moreover, the study indicates that the use of sodium nitrate statistically has no contribution to the stability of graphene oxide dispersion statistically. There was no statistical difference which was found in terms of the dispersion's stability between the improved Hummers method which doesn't give an opportunity to the release of NOx gases environmentally with the classical Hummers method which include the sodium nitrate. The improved Hummers method is an important option especially in order that NOx gases don't release to the environment. Especially, this subject will gain much more importance in the large-scaled industrial generations. It is an important reason that the experiment-friendly improved method is chosen from the methods which don't have the statistical difference in terms of the dispersion stability. Moreover, the phosphoric acid is a more-easily accessible and cheaper material than the sodium nitrate when the production cost is considered. The method's advantage has been added to the article in consideration with the reviewer's comment.

Thus, the improved Hummers method should be used in order to obtain the graphene oxide dispersions (for the more stable dispersions) in the demanded properties. The reduction performance of sodium borohydride and ascorbic acid was tested statistically with the same mixture ratios. There was no statistical difference which was found in terms of the dispersion stability between these two reducers. Thus, it was concluded that the ascorbic acid may be preferred the in the phase of reduction for more environmentally friendly production.

The improvement rate of the quality characteristic between the estimate and the optimal conditions were calculated for GO dispersions as the following; average particle size, $264.0 \%$, the standard deviation of particle size, $1875.0 \%$, the polydispersity index, $583.3 \%$, the zeta potential, $5.0 \%$ and the conductivity, $50.0 \%$. Also, it can be seen from the results of the produced graphene oxide dispersions that they satisfied the expected properties. The improvement rate of the quality characteristic between the estimate and the optimal conditions were calculated for rGO dispersions as the following; average particle size, $42.7 \%$, the standard deviation of particle size, $79.7 \%$, the polydispersity index, $-5.0 \%$, the zeta potential, $9.9 \%$ and the conductivity, $-86.7 \%$. Also, it can be seen from the results of the produced reduced graphene oxide samples that they satisfied the expected properties.

The results show that the proposed methodology in this study is effective to improve graphene oxide dispersion stability. Also, this methodology is easy to adaptable the other graphene based material synthesis process such as Brodie, improved Hummers methodology or chemical vapor deposition method.

\section{References}

[1] Hu, X., Yu, Y., Wang, Y., Zhou, J., Song, L. "Separating nano graphene oxide from the residual strong-acid filtrate of the modified Hummers method with alkaline solutio." Applied Surface Science. 329, pp. 83-86. 2015 .

https://doi.org/10.1016/j.apsusc.2014.12.110

[2] Guerrero-Contreras, J., Caballero-Briones, F. "Graphene oxide powders with different oxidation degree, prepared by synthesis variations of the Hummers method." Materials Chemistry and Physics. 153, pp. 209-220. 2015 .

https://doi.org/10.1016/j.matchemphys.2015.01.005

[3] Chen, L., Xu, C., Liu, J., Fang, X., Zhang, Z. "Optical absorption property and photo-thermal conversion performance of graphene oxide/water nanofluids with excellent dispersion stability." Solar Energy. 148, pp. 17-24. 2017.

https://doi.org/10.1016/j.solener.2017.03.073

[4] Zhang, C., Zhang, L., Xu, H., Wang, D., Ye, B. "Investigation of flow boiling performance and the resulting surface deposition of graphene oxide nanofluid in microchannels." Experimental Thermal and Fluid Science. 86, pp. 1-10. 2017.

https://doi.org/10.1016/j.expthermflusci.2017.03.028

[5] Nagavolu, C., Susmitha, K., Raghavender, M., Giribabu, L., Bhanu Sankara Rao, K., Smith, C. T. G., Mills, C. A., Silva, S. R. P., Srikanth, V. V. S. S. "Pt-free spray coated reduced graphene oxide counter electrodes for dye sensitized solar cells." Solar Energy. 137, pp. 143-147. 2016. https://doi.org/10.1016/j.solener.2016.08.002

[6] Belekoukia, M., Ploumistos, A., Sygellou, L., Nouri, E., Tasis, D., Lianos, P. "Co-N doped reduced graphene oxide used as efficient electrocatalyst for dye-sensitized solar cells." Solar Energy Materials and Solar Cells. 157, pp. 591-598. 2016.

https://doi.org/10.1016/j.solmat.2016.07.042

[7] Perumal, S., Lee, H. M., Cheong, I. W. "High-concentration graphene dispersion stabilized by block copolymers in ethanol." Journal of Colloid and Interface Science. 497, pp. 359-367. 2017.

https://doi.org/10.1016/j.jcis.2017.03.027

[8] Li, X., Korayem, A. H., Li, C., Liu, Y., He, H., Sanjayan, J. G., Duan, W. H. "Incorporation of graphene oxide and silica fume into cement paste: A study of dispersion and compressive strength." Construction and Building Materials. 123, pp. 327-335. 2016. https://doi.org/10.1016/j.conbuildmat.2016.07.022

[9] Fan, L., Ge, H., Zou, S., Xiao, Y., Wen, H., Li, Y., Feng, H., Nie, M. "Sodium alginate conjugated graphene oxide as a new carrier for drug delivery system." International Journal of Biological Macromolecules. 93(Part A), pp. 582-590. 2016. https://doi.org/10.1016/j.ijbiomac.2016.09.026

[10] Bai, M., Chen, J., Wu, W., Zeng, X., Wang, J., Zou, H. "Preparation of stable aqueous dispersion of edge-oxidized graphene and its transparent conductive films." Colloids and Surfaces A: Physicochemical and Engineering Aspects. 490, pp. 59-66. 2016. https://doi.org/10.1016/j.colsurfa.2015.11.033

[11] Yang, S., Li, G., Wang, D., Qiao, Z., Qu, L. "Synthesis of nanoneedle-like copper oxide on N-doped reduced graphene oxide: A three-dimensional hybrid for nonenzymatic glucose sensor." Sensors and Actuators B: Chemical. 238, pp. 588-595. 2017. https://doi.org/10.1016/j.snb.2016.07.105

[12] Oyedotun, K. O., Madito, M. J., Bello, A., Momodu, D. Y., Mirghni, A. A., Manyala, N. "Investigation of graphene oxide nanogel and carbon nanorods as electrode for electrochemical supercapacitor." Electrochimica Acta. 245, pp. 268-278. 2017.

https://doi.org/10.1016/j.electacta.2017.05.150 
[13] Tabrizi, A. G., Arsalani, N., Namazi, H., Ahadzadeh, I. "Vanadium oxide assisted synthesis of polyaniline nanoarrays on graphene oxide sheets and its application in supercapacitors." Journal of Electroanalytical Chemistry. 798, pp. 34-41. 2017. https://doi.org/10.1016/j.jelechem.2017.04.059

[14] Fathalipour, S., Mardi, M. "Synthesis of silane ligand-modified graphene oxide and antibacterial activity of modified graphene-silver nanocomposite." Materials Science and Engineering: C. 79, pp. 55-65. 2017. https://doi.org/10.1016/j.msec.2017.05.020

[15] Ji, X., Song, Y., Han, J., Ge, L., Zhao, X., Xu, C., Wang, Y., Wu, D., Qiu, H. "Preparation of a stable aqueous suspension of reduced graphene oxide by a green method for applications in biomaterials." Journal of Colloid and Interface Science. 497, pp. 317-324. 2017. https://doi.org/10.1016/j.jcis.2016.09.049

[16] Yoo, S., Lee, J., Kim, J. M., Seong, C.-Y., Seong, K.-d., Piao, Y. "Well-dispersed sulfur wrapped in reduced graphene oxide nanoscroll as cathode material for lithium-sulfur battery." Journal of Electroanalytical Chemistry. 780, pp. 19-25. 2016.

https://doi.org/10.1016/j.jelechem.2016.08.040

[17] Lingamdinne, L. P., Koduru, J. R., Roh, H., Choi, Y.-L., Chang, Y.-Y., Yang, J.-K. "Adsorption removal of Co(II) from waste-water using graphene oxide." Hydrometallurgy. 165(Part 1), pp. 90-96. 2016. https://doi.org/10.1016/j.hydromet.2015.10.021

[18] Liu, Y., Zhang, Y., Duan, L., Zhang, W., Su, M., Sun, Z., He, P. "Polystyrene/graphene oxide nanocomposites synthesized via Pickering polymerization." Progress in Organic Coatings. 99, pp. 23-31. 2016. https://doi.org/10.1016/j.porgcoat.2016.04.034

[19] Zhao, Y., Lu, J., Liu, X., Wang, Y., Lin, J., Peng, N., Li, J., Zhao, F. "Performance enhancement of polyvinyl chloride ultrafiltration membrane modified with graphene oxide." Journal of Colloid and Interface Science. 480, pp. 1-8. 2016.

https://doi.org/10.1016/j.jcis.2016.06.075

[20] Michel, M., Biswas, C., Kaul, A. B. "High-performance ink-jet printed graphene resistors formed with environmentally-friendly surfactant-free inks for extreme thermal environments." Applied Materials Today. 6, pp. 16-21. 2017.

https://doi.org/10.1016/j.apmt.2016.12.001

[21] Konios, D., Stylianakis, M. M., Stratakis, E., Kymakis, E. "Dispersion behaviour of graphene oxide and reduced graphene oxide." Journal of Colloid and Interface Science. 430, pp. 108-112. 2014. https://doi.org/10.1016/j.jcis.2014.05.033

[22] Taha-Tijerina, J., Venkataramani, D., Aichele, C. P., Tiwary, C. S., Smay, J. E., Mathkar, A., Chang, P., Ajayan, P. M. "Quantification of the Particle Size and Stability of Graphene Oxide in a Variety of Solvents." Particle \& Particle Systems Characterization. 32(3), pp. 334-339. 2015. https://doi.org/10.1002/ppsc.201400099

[23] Esfahani, M. R., Languri, E. M., Nunna, M. R. "Effect of particle size and viscosity on thermal conductivity enhancement of graphene oxide nanofluid." International Communications in Heat and Mass Transfer. 76, pp. 308-315. 2016.

https://doi.org/10.1016/j.icheatmasstransfer.2016.06.006

[24] Hu, X., Yu, Y., Hou, W., Zhou, J., Song, L. "Effects of particle size and $\mathrm{pH}$ value on the hydrophilicity of graphene oxide." Applied Surface Science. 273, pp. 118-121. 2013.

https://doi.org/10.1016/j.apsusc.2013.01.201

[25] Shu, R., Yin, Q., Xing, H., Tan, D., Gan, Y., Xu, G. "Colloidal and rheological behavior of aqueous graphene oxide dispersions in the presence of poly(ethylene glycol)." Colloids and Surfaces A: Physicochemical and Engineering Aspects. 488, pp. 154-161. 2016.

https://doi.org/10.1016/j.colsurfa.2015.10.006
[26] Johnson, D. W., Dobson, B. P., Coleman, K. S. "A manufacturing perspective on graphene dispersions." Current Opinion in Colloid \& Interface Science. 20(5), pp. 367-382. 2015.

https://doi.org/10.1016/j.cocis.2015.11.004

[27] Zhang, H., Wang, S., Lin, Y., Feng, M., Wu, Q. "Stability, thermal conductivity, and rheological properties of controlled reduced graphene oxide dispersed nanofluids." Applied Thermal Engineering. 119, pp. 132-139. 2017. https://doi.org/10.1016/j.applthermaleng.2017.03.064

[28] Hajjar, Z., Rashidi, A. M., Ghozatloo, A. "Enhanced thermal conductivities of graphene oxide nanofluids." International Communications in Heat and Mass Transfer. 57, pp. 128-131. 2014.

https://doi.org/10.1016/j.icheatmasstransfer.2014.07.018

[29] Kim, K. M., Bang, I. C. "Effects of graphene oxide nanofluids on heat pipe performance and capillary limits." International Journal of Thermal Sciences. 100, pp. 346-356. 2016.

https://doi.org/10.1016/j.ijthermalsci.2015.10.015

[30] Obreja, A. C., Cristea, D., Gavrila, R., Schiopu, V., Dinescu, A., Danila, M., Comanescu, F. "Isocyanate functionalized graphene/P3HT based nanocomposites." Applied Surface Science. 276, pp. 458-467. 2013. https://doi.org/10.1016/j.apsusc.2013.03.117

[31] Sohail, M., Saleem, M., Ullah, S., Saeed, N., Afridi, A., Khan, M. "Modified and improved Hummer's synthesis of graphene oxide for capacitors applications." Modern Electronic Materials. 3(3), pp. 110-116. 2017. https://doi.org/10.1016/j.moem.2017.07.002

[32] Chen, J., Li, Y., Huang, L., Li, C., Shi, G. "High-yield preparation of graphene oxide from small graphite flakes via an improved Hummers method with a simple purification process." Carbon. 81, pp. 826-834. 2015. https://doi.org/10.1016/j.carbon.2014.10.033

[33] Gupta, V., Sharma, N., Singh, U., Arif, M., Singh, A. "Higher oxidation level in graphene oxide." Optik - International Journal for Light and Electron Optics. 143(Supplement C), pp. 115-124. 2017. https://doi.org/10.1016/j.ijleo.2017.05.100

[34] Huang, M.-L., Hung, Y.-H., Yang, Z.-S. "Validation of a method using Taguchi, response surface, neural network, and genetic algorithm." Measurement. 94, pp. 284-294. 2016.

https://doi.org/10.1016/j.measurement.2016.08.006

[35] Debnath, S., Reddy, M. M., Yi, Q. S. "Influence of cutting fluid conditions and cutting parameters on surface roughness and tool wear in turning process using Taguchi method." Measurement. 78, pp. 111-119. 2016. https://doi.org/10.1016/j.measurement.2015.09.011

[36] Box, G. E. P., Hunter, J. S., Hunter, W. G. "Statistics for Experimenters: Design, Innovation, and Discovery." 2nd edition, John Wiley \& Sons. 2005.

[37] Bilga, P. S., Singh, S., Kumar, R. "Optimization of energy consumption response parameters for turning operation using Taguchi method." Journal of Cleaner Production. 137, pp. 1406-1417. 2016 https://doi.org/10.1016/j.jclepro.2016.07.220

[38] Mitra, A. C., Jawarkar, M., Soni, T., Kiranchand, G. R. "Implementation of Taguchi Method for Robust Suspension Design." Procedia Engineering. 144, pp. 77-84. 2016.

https://doi.org/10.1016/j.proeng.2016.05.009

[39] Şimşek, B., İç, Y. T., Şimşek, E. H. "A RSM-Based Multi-Response Optimization Application for Determining Optimal Mix Proportions of Standard Ready-Mixed Concrete." Arabian Journal for Science and Engineering. 41(4), pp. 1435-1450. 2016.

https://doi.org/10.1007/s13369-015-1987-0

[40] Şimşek, B., İç, Y. T., Şimşek, E. H. "A TOPSIS-based Taguchi optimization to determine optimal mixture proportions of the high strength self-compacting concrete." Chemometrics and Intelligent Laboratory Systems. 125, pp. 18-32. 2013.

https://doi.org/10.1016/j.chemolab.2013.03.012 
[41] Jang, G. G., Song, B., Moon, K.-S., Wong, C.-P., Keum, J. K., Hu, M. Z. "Particle size effect in porous film electrodes of ligand-modified graphene for enhanced supercapacitor performance." Carbon. 119, pp. 296-304. 2017.

https://doi.org/10.1016/j.carbon.2017.04.023

[42] Dong, L., Li, M., Dong, L., Zhao, M., Feng, J., Han, Y., Deng, J., Li, X., Li, D., Sun, X. "Hydrothermal synthesis of mixed crystal phases TiO2reduced graphene oxide nanocomposites with small particle size for lithium ion batteries." International Journal of Hydrogen Energy. 39(28), pp. 16116-16122. 2014.

https://doi.org/10.1016/j.ijhydene.2014.01.029

[43] Sawtarie, N., Cai, Y., Lapitsky, Y. "Preparation of chitosan/tripolyphosphate nanoparticles with highly tunable size and low polydispersity." Colloids and Surfaces B: Biointerfaces. 157, pp. 110-117. 2017. https://doi.org/10.1016/j.colsurfb.2017.05.055

[44] Konkena, B., Vasudevan, S. "Understanding Aqueous Dispersibility of Graphene Oxide and Reduced Graphene Oxide through pKa Measurements." The Journal of Physical Chemistry Letters. 3(7), pp. 867-872. 2012. https://doi.org/10.1021/jz300236w

[45] Kim, J., Kwon, S., Cho, D.-H., Kang, B., Kwon, H., Kim, Y., Park, S. O., Jung, G. Y., Shin, E., Kim, W.-G., Lee, H., Ryu, G. H., Choi, M., Kim, T. H., Oh, J., Park, S., Kwak, S. K., Yoon, S. W., Byun, D., Lee, Z., Lee, C. "Direct exfoliation and dispersion of two-dimensional materials in pure water via temperature control." Nature Communications. 6, 8294. 2015. https://doi.org/10.1038/ncomms9294

[46] Majee, S., Liu, C., Wu, B., Zhang, S. L., Zhang, Z. B. "Ink-jet printed highly conductive pristine graphene patterns achieved with water-based ink and aqueous doping processing. Carbon. 114, pp. 77-83. 2017. https://doi.org/10.1016/j.carbon.2016.12.003

[47] Botas, C., Álvarez, P., Blanco, P., Granda, M., Blanco, C., Santamaría, R., Romasanta, L. J., Verdejo, R., López-Manchado, M. A., Menéndez, R. "Graphene materials with different structures prepared from the same graphite by the Hummers and Brodie methods." Carbon. 65, pp. 156164. 2013

https://doi.org/10.1016/j.carbon.2013.08.009

[48] Fathy, M., Gomaa, A., Taher, F. A., El-Fass, M. M., Kashyout, A. E.-H. B. "Optimizing the preparation parameters of $\mathrm{GO}$ and $\mathrm{rGO}$ for large-scale production." Journal of Materials Science. 51(12), pp. 5664-5675. 2016. https://doi.org/10.1007/s10853-016-9869-8

[49] Kondratowicz, I., Żelechowska, K., Sadowski, W. "Optimization of Graphene Oxide Synthesis and Its Reduction." In: Fesenko, O., Yatsenko, L. (eds.), Nanoplasmonics, Nano-Optics, Nanocomposites, and Surface Studies: Selected Proceedings of the Second FP7 Conference and the Third International Summer School Nanotechnology: From Fundamental Research to Innovations, August 23-30, 2014, Yaremche-Lviv, Ukraine. (pp. 467-484). Cham: Springer International Publishing. 2015.

[50] Krishnamoorthy, K., Veerapandian, M., Yun, K., Kim, S. J. "The chemical and structural analysis of graphene oxide with different degrees of oxidation." Carbon. 53, pp. 38-49. 2013. https://doi.org/10.1016/j.carbon.2012.10.013

[51] Poorali, M.-S., Bagheri-Mohagheghi, M.-M. "Comparison of chemical and physical reduction methods to prepare layered graphene by graphene oxide: optimization of the structural properties and tuning of energy band gap." Journal of Materials Science: Materials in Electronics. 27(1), pp. 260-271. 2016.

https://oi.org/10.1007/s10854-015-3749-x

[52] Shahriary, L., Athawale, A. A. "Graphene oxide synthesized by using modified hummers approach." International Journal of Renewable Energy and Environmental Engineering. 2, pp. 58-63. 2014.
[53] Yong Jae, K., Yung Ho, K., Yun-Hwa, H., Sun Min, L., Sung-Youp, L., Hyeong-Rag, L., Seoung Ho, L., Sang Hoon, N., Won Bae, K., Kwanghee, L. "Optimization of graphene oxide synthesis parameters for improving their after-reduction material performance in functional electrodes." Materials Research Express. 3(10), 105033. 2016. [Online]. Availavle from: http://stacks.iop.org/2053-1591/3/i=10/a=105033 [Accessed: 1st April 2017]

[54] Rattana, Chaiyakun, S., Witit-anun, N., Nuntawong, N., Chindaudom, P., Oaew, S., Kedkeaw, C., Limsuwan, P. "Preparation and characterization of graphene oxide nanosheets." Procedia Engineering, 32, pp. 759-764. 2012.

https://doi.org/10.1016/j.proeng.2012.02.009

[55] Sudesh, Kumar, N., Das, S., Bernhard, C., Varma, G. D. "Effect of graphene oxide doping on superconducting properties of bulk $\mathrm{MgB}$ 2." Superconductor Science and Technology. 26(9), 095008. 2013. [Online]. Availavle from: http://stacks.iop.org/0953-2048/26/i=9/a=095008 [Accessed: 1st April 2017]

[56] Mahmood, H., Habib, A., Mujahid, M., Tanveer, M., Javed, S., Jamil, A. "Band gap reduction of titania thin films using graphene nanosheets." Materials Science in Semiconductor Processing. 24, pp. 193-199. 2014. https://doi.org/10.1016/j.mssp.2014.03.038

[57] Muhammad Hafiz, S., Ritikos, R., Whitcher, T. J., Md. Razib, N., Bien, D. C. S., Chanlek, N., Nakajima, H., Saisopa, T., Songsiriritthigul, P., Huang, N. M., Rahman, S. A. "A practical carbon dioxide gas sensor using room-temperature hydrogen plasma reduced graphene oxide." Sensors and Actuators B: Chemical. 193, pp. 692-700. 2014. https://doi.org/10.1016/j.snb.2013.12.017

[58] Zhang, X., Li, K., Li, H., Lu, J., Fu, Q., Chu, Y. "Graphene nanosheets synthesis via chemical reduction of graphene oxide using sodium acetate trihydrate solution." Synthetic Metals. 193, pp. 132-138. 2014. https://doi.org/10.1016/j.synthmet.2014.04.007

[59] Hou, D., Liu, Q., Cheng, H., Zhang, H., Wang, S. "Green reduction of graphene oxide via Lycium barbarum extract." Journal of Solid State Chemistry. 246, pp. 351-356.

https://doi.org/10.1016/j.jssc.2016.12.008

[60] Tansel İç, Y. "Development of a credit limit allocation model for banks using an integrated Fuzzy TOPSIS and linear programming." Expert Systems with Applications. 39(5), pp. 5309-5316. 2012.

https://doi.org/10.1016/j.eswa.2011.11.005

[61] Şimşek, B., Uygunoğlu, T. "Multi-response optimization of polymer blended concrete: A TOPSIS based Taguchi application." Construction and Building Materials. 117, pp. 251-262. 2016.

https://doi.org/10.1016/j.conbuildmat.2016.05.027

[62] De Silva, K. K. H., Huang, H. H., Joshi, R. K., Yoshimura, M. "Chemical reduction of graphene oxide using green reductants." Carbon. 119(Supplement C), pp. 190-199. 2017.

https://doi.org/10.1016/j.carbon.2017.04.025

[63] Liu, S., Zeng, T. H., Hofmann, M., Burcombe, E., Wei, J., Jiang, R., Kong, J., Chen, Y. "Antibacterial Activity of Graphite, Graphite Oxide, Graphene Oxide, and Reduced Graphene Oxide: Membrane and Oxidative Stress." ACS Nano. 5(9), pp. 6971-6980. 2011. https://doi.org/10.1021/nn202451x

[64] Šimšíková, M. "Interaction of graphene oxide with albumins: Effect of size, $\mathrm{pH}$, and temperature." Archives of Biochemistry and Biophysics. 593, pp. 69-79. 2016. https://doi.org/10.1016/j.abb.2016.02.015 